\title{
REVIEWS
}

\section{PET/CT Imaging for Monitoring Recurrence and Evaluating Response to Treatment in Breast Cancer}

\author{
Department of Chemotherapy Center, Zhejiang Cancer Hospital, Hangzhou, Zhejiang, China
}

A - research concept and design; $\mathbf{B}$ - collection and/or assembly of data; $\mathbf{C}$ - data analysis and interpretation;

$\mathbf{D}$ - writing the article; $\mathbf{E}$ - critical revision of the article; $\mathbf{F}$ - final approval of article

\begin{abstract}
Monitoring recurrence and evaluating response to therapy are important aspects of clinical decision making in the treatment of breast cancer. In this literature review, the authors highlight several of the key areas where integrated fluorine-18 fluorodeoxyglucose positron emission tomography/computed tomography (FDG PET/CT) measurements are anticipated to have a significant impact on monitoring recurrence and evaluating response to therapy. These areas include comparing FDG PET/CT with conventional imaging for detecting breast cancer metastases; evaluating the role of FDG PET/CT in the presence of elevated tumor markers during follow-up period after the primary surgery; using FDG PET/CT to assess response to neoadjuvant chemotherapy (NAC), targeted and endocrine therapies; using FDG PET/CT to predict response to NAC according to different molecular phenotypes of breast cancer; and applying PET/CT and some new breast-related PET tracers to evaluate response to anticancer treatment. The authors consider the relative advantages afforded by PET/CT and summarize current evidence as to the likely value of PET/CT in recurrence detection and anticancer treatment response prediction (Adv Clin Exp Med 2016, 25, 2, 377-382).
\end{abstract}

Key words: breast cancer, FDG PET, treatment monitoring.

Breast cancer is the most frequently diagnosed form of cancer and the leading cause of cancer death in women worldwide. The first commercial positron emission tomography/computed tomography (PET/CT) unit was introduced in 2000, as a noninvasive, one-stop evaluation, merging anatomic localization with a functional image that enables measurements of physiological function in the region scanned. So far, the most useful application of PET/CT has been in monitoring breast cancer recurrence and in evaluating response to therapy by detecting changes in $18 \mathrm{~F}$-fluorodeoxyglucose (18F-FDG) uptake. In this paper, current applications of PET/CT in the management of breast cancer are summarized.

\section{Monitoring Recurrence}

Under standard protocols, following treatment for their initial breast cancer, patients are routinely followed up with clinical examinations and mammography for at least five years. Recurrence or metastases usually happen in the first two to three years. Recurrence may be localized (in the breast), regional (affecting lymph nodes in the ipsilateral axilla), or cancer may recur as distant metastases (in tissues such as bone, liver, lungs or brain). One study by Elder et al. showed that the median interval from primary surgery to recurrence was 2.3 years; $27 \%$ of 456 patients with recurrence had bone metastases, $27 \%$ had local recurrence, $16 \%$ had lung metastases and $13 \%$ had liver metastases [1]. Current strategies for the detection of breast cancer recurrence include computed tomography (CT), magnetic resonance imaging (MRI), ultrasound (US) and bone scintigraphy (BS). In addition to conventional technologies, PET/CT has recently been shown to have relevance in the detection and management of breast cancer recurrence.

In breast cancer patients, accurate restaging of recurrence after systematic therapy is crucial 
for the selection of the most appropriate therapeutic strategy. In comparison with conventional methods, such as US, CT, BS and mammography, PET/CT has been shown to have superior specificity and sensitivity in the detection of distant metastases [2]. False negative PET/CT results can occur because of low FDG uptake in some conditions, such as invasive lobular carcinoma, less well visualized lesions in the visceral cavity and poor imaging of the limbs and brain. False positive results, on the other hand, may occur in inflammations. In a meta-analysis by Pennant et al. [3], PET/ /CT had significantly higher accuracy as compared with CT or PET alone for the diagnosis of recurrent breast cancer. No significant differences were found in the sensitivity or specificity of PET (with or without CT) when compared with MRI [3-4]. Since PET/CT and MRI have shown considerable effective in the verification of breast cancer recurrence or metastases, PET/MRI may become a powerful and promising technique in clinical oncology in the near future.

Schmidt et al. found PET/CT to be more sensitive than whole body-MRI (WB-MRI) in detecting lymph node involvement [4]. In addition, PET-CT detected more osteolytic-type bone lesions than osteoblastic lesions as compared with BS [5]. One possible reason for this difference may be that osteoblast proliferation in osteosclerotic lesions increases the bone matrix, where FDG uptake is lower. Osteoblastic metastases usually do not show increased glucose intake and are better detected with BS.

Evangelista et al. carried out a systematic review involving 13 studies from 2001 to May 2011, which showed that fluorodeoxyglucose PET, and in particular PET/CT, allows $83 \%$ accuracy in the assessment of the site and extent of the recurring disease when the clinician is confronted with suspicious elevated serum tumor markers in asymptomatic breast cancer patients following primary treatment and negative conventional imaging findings [6].

In breast cancer patients suspected of disease recurrence, CA15-3 levels, as well as increases in CA15-3 levels over time, have proven systematically higher in PET-positive patients when compared with PET-negative patients. However, no single cut-off value yielding an acceptable sensitivity and specificity has been identified for either variable. Rather, it is the combination of increases in CA15-3 over time along with a clinical suspicion of disease progression that should prompt the clinician to perform PET/CT imaging. The limited data available further suggests that this approach will likely affect treatment management in up to $50 \%$ of patients [7].
Evangelista et al. retrospectively studied 60 breast cancer patients who had already undergone systemic therapy for primary disease. Three serial measures of Ca15-3 were collected individually within one year before PET/CT examination: at 12-9 months, at 9-3 months, and at 3-0 months. The study showed that an increase in Ca15-3 at 9-3 months and 3-0 months correlates with accurate positive PET/CT results and disease relapse $(\mathrm{p}<0.05)$.During a breast cancer patient's follow-up, serial elevated Ca15-3 could be a hint to the clinician to perform a PET/CT examination to detect disease relapse when the disease is more treatable [8].

These findings indicate that PET/CT is an accurate, sensitive and reliable modality for the screening and detection of breast cancer recurrence. According to the 2014 National Comprehensive Cancer Network guidelines (NCCN 2014 v. 3) FDG PET/CT is highly recommended for the detection of suspicious or equivocal metastatic lesions and bone metastasis [9].

FDG-PET/CT appears to be an effective surveillance tool; the technique is able to assess the whole body in a single procedure and performs well. More prospective studies are needed to determine whether this technology could potentially replace conventional imaging tests used today to monitor breast cancer recurrence.

\section{Assessment of Response to Therapy}

Neoadjuvant chemotherapy (NAC) has long been a standard therapy for stage II and III breast cancer [10]. It is generally accepted that an absence of residual cancer cells in the primary tumor following NAC is strongly associated with improved disease-free survival and overall survival [11]. However, in most NAC studies less than $30 \%$ of patients achieve complete response [12]. Thus, find an effective way to predict response early during NAC is important so that physicians can change strategies in case of ineffectiveness, thereby avoiding unnecessary side effects to patients.

A correlation has been demonstrated between early changes in the maximum standardized uptake value (after one or two courses of chemotherapy) and the final treatment response at the completion of NAC [13-15]. Relative changes in both the maximum standardized uptake value (SUV -max) and standardized uptake value (SUV) have been proposed as a means to discriminate metabolic responders from nonresponders or to differentiate between pathological complete response (pCR) and non-pCR [13-15]. Recently, a study by 
Andrade et al. demonstrated that the optimal percentage change in post-treatment SUV scores relative to baseline ( $\triangle \mathrm{SUV})$ to discriminates between pCR and non-pCR was $-71.8 \%$ (83.3\% sensitivity; $78.5 \%$ specificity); the optimal $\triangle$ SUV threshold to discriminate between NAC responders and non-responders was - 59.1\% (68\% sensitivity; $75.0 \%$ specificity) [16]. Unfortunately, the optimal threshold value varies dramatically across studies.

There are still studies suggesting that other imaging tests, such as MRI, when combined with tumor diameter detection, are better than the change in SUV for evaluating response to NAC [17]. However, in general more and more data support the role of FDG PET/CT in the early evaluation of response to NAC. Better defined criteria for PET/CT evaluation are needed. MRI is also useful in the early evaluation of response to NAC, and the place of PET/CT in comparison with MRI needs to be better determined [18].

It is possible that PET/CT can detect tumor response metabolically earlier than morphologic imaging methods in order to evaluate treatment response in metastatic breast cancer. First, for targeted therapies, tumors always change metabolically earlier than their size changes. Second, for endocrine therapy, several studies have shown that an increase in tumor FDG uptake 7-10 days after initiating endocrine therapy is predictive of a good response [19-20]. This phenomenon can be explained by the fact that endocrine therapy has initial agonist effects before antagonist effects dominate. Therefore, an increase in SUV in tumors soon after the initiation of hormone therapy is predictive of a good therapeutic response. One study by Mortazavi-Jehanno et al. found that "metabolic response assessed by FDG PET/CT imaging in metastatic breast cancer treated with endocrine therapy is predictive of the patients' (progression-free survival)" [21]. However, data are lacking on the delayed effect of endocrine therapy on tumor metabolism. Third, PET/CT is also helpful in providing evidence of a heterogeneous response (that is, the co-existence of responding and non-responding lesions within the same patient) [22].

Newer evaluation criteria are needed that take into account not only the PET but also the CT of hybrid imaging. Although there are few studies evaluating PET/CT in metastatic breast cancer treatment, this modality does have some advantages over other imaging. More data are needed, and standardization in the criteria for evaluation is necessary.

Previous research has evaluated correlations between the difference in SUV in dual time-point imaging and biological prognostic factors [23-25]. Little is known, however, as to whether the meta- bolic characteristics of breast cancer differ in relation to molecular subtypes. Triple-negative breast tumors (negative for estrogen and progesterone receptors, and no HER2/neu overexpression) are currently the subject of major interest because of the aggressiveness of these tumors, their poor prognosis and the lack of targeted therapy. Some studies focusing on triple-negative breast cancer (TNBC) have shown that the SUV of the primary lesion in the TNBC group was significantly higher than in non-TNBC $[23,26]$. One study by Keam et al. showed that the estrogen receptor-negative phenotype had a higher percentage change in SUV and pre-chemotherapy SUV than other phenotypes [26]. Non-TNBC has lower pre-chemotherapy SUV than TNBC patients (9.8 vs. 6.4, $\mathrm{p}=0.008$ ) [25]. Early metabolic non-response was always associated with pathologic non-response and a poor prognosis in ER-positive/HER2-negative patients. In this subtype, PET/CT might be useful to select patients who will probably benefit from early therapeutic strategy modifications $[27,28]$.

Recently, Koolen et al. evaluated the effect of $18 \mathrm{~F}-\mathrm{FDG} \mathrm{PET} / \mathrm{CT}$ in monitoring responses to NAC in 98 breast cancer patients. They found that the accuracy of response monitoring with PET/ /CT was much higher in the ER-positive/HER2-negative and triple-negative subtypes than in the HER2-positive subtype. They speculated that this phenomenon could be explained by an initial inflammatory response induced by trastuzumab treatment in HER2-positive subtype patients [29].

There are other oncologic PET-tracers for the management of breast cancer. PET with 18F-FLT enables investigators to quantify the proliferation fraction of tumors [30] and has been proposed as a better biomarker than FDG-PET for measuring response to therapy [31]. In a study of 14 breast cancer patients, FDG-PET and FLT-PET were performed at baseline, two weeks after the first cycle of chemotherapy or endocrine therapy and after the completion of treatment [32]. Early changes in FLT uptake showed a stronger correlation with clinical outcome than did FDG. According to preliminary results from a pilot study, changes in the number of circulatory tumor cells (CTCs) seem to be correlated with changes in FLT-PET uptake in metastatic lesions during the course of therapy [33]. Larger prospective trials are needed to define the potential role of FLT-PET for treatment monitoring.

Another biomarker, 16a-18F-fluoro-17b-estradiol (FES), is a steroid-based positron emission tomography tracer that has been shown to be a reliable tracer for the management of ER-positive breast cancer patients. Low FES uptake in tumor lesions shows a strong predictive value for failure of antihormonal therapy [34]. Yet another bio- 
marker, 21-[F-18]-fluoro-16a,17a-[(R)-(19-a-furyl methylidene)dioxy]-19-norpregn-4-ene-3,20-dione (FFNP), shows a high affinity and selectivity for progesterone receptors (PR) [35]. In the future, FFNP-PET may allow for the non-invasive detection and quantification of PR-positive lesions.

[Zr89]-trastuzumab, which shows an excellent uptake in HER2-positive lesions in liver, lung, bone and brain metastases, may allow for a whole-body assessment of the HER2 receptor status in metastatic/recurrent breast cancer. However, so far few reports have described the use of this biomarker in breast cancer patients [36].

Piccardo et al. found that (18) F-Fluoride$\mathrm{PET} /$ multi-detector row spiral CT (MDCT) with $1.25 \mathrm{~mm}$ section thickness image reconstruction has a higher diagnostic accuracy for bone metastases than does PET/CT and MDCT [37].

It has also recently been argued that $18 \mathrm{~F}$-sodium fluoride (18F-NaF) PET/CT might prove a useful alternative to detect bone reaction to metastatic involvement. Combined NaF/FDG-PET/CT has shown promising early results using the same amount of radiation as BS and FDG-PET/CT [38]. According to Gradishar et al., if a FDG PET/CT scan clearly indicates bone metastasis on both the PET and CT components, a bone scan or sodium fluoide PET/CT may not be needed [9]. In some conditions, considering the coexistence of both lytic and sclerotic lesions and the different mechanisms of radiotracer uptake, PET and BS are mutually complementary methods for the diagnosis of bone metastasis [38].

\section{Conclusions}

Studies to date support the following conclusions: 1) PET/CT can be used earlier than morphologic imaging methods to evaluate the response to chemotherapy, targeted therapy and endocrine treatment in breast cancer. 2) Serial elevated Ca15-3 could be a hint to the clinician to perform a PET/CT examination to detect disease relapse when the disease is at a more treatable stage. 3) TNBC was significantly associated with a higher SUV (max) and relatively good response to NAC. 4) New PET biomarkers, including those that detect the rate of cell proliferation and the expression of estrogen receptors, seek to improve patients' restaging information and the evaluation of therapeutic effectiveness. Further studies are needed to evaluate these new imaging strategies and their clinical utility. It is likely that in the future those patients who are unlikely to benefit from chemotherapy could be identified by a pretreatment PET/CT test.

\section{References}

[1] Elder EE, Kennedy CW, Gluch L, Carmalt HL, Janu NC, Joseph MG, Donellan MJ, Molland JG, Gillett DJ: Patterns of breast cancer relapse. Eur J Surg Oncol 2006, 32, 922-927.

[2] Niikura N, Costelloe CM, Madewell JE, Hayashi N, Yu TK, Liu J, Palla SL, Tokuda Y, Theriault RL, Hortobagyi GN, Ueno NT: FDG-PET/CT compared with conventional imaging in the detection of distant metastases of primary breast cancer. The Oncologist 2011, 16, 1111-1119.

[3] Pennant M, Takwoingi Y, Pennant L, Davenport C, Fry-Smith A, Eisinga A, Andronis L, Arvanitis T, Deeks J, Hyde C: A systematic review of positron emission tomography (PET) and positron emission tomography/computed tomography (PET/CT) for the diagnosis of breast cancer recurrence. Health Technol Assess 2010, 14, 1-103.

[4] Schmidt GP, Baur-Melnyk A, Haug A, Heinemann V, Bauerfeind I, Reiser MF, Schoenberg SO: Comprehensive imaging of tumor recurrence in breast cancer patients using whole-body MRI at 1.5 and 3 T compared to FDG-PET-CT. Euro J Radiol 2008, 65, 47-58.

[5] Constantinidou A, Martin A, Sharma B, Johnston SR: Positron emission tomography/computed tomography in the management of recurrent/metastatic breast cancer: a large retrospective study from the Royal Marsden Hospital. Ann Oncol 2011, 22, 307-314.

[6] Evangelista L, Cervino AR, Ghiotto C, Al-Nahhas A, Rubello D, Muzzio PC: Tumor marker-guided PET in breast cancer patients-a recipe for a perfect wedding: a systematic literature review and meta-analysis. Clin Nucl Med 2012, 37, 467-474.

[7] Filippi V, Malamitsi J, Vlachou F, Laspas F, Georgiou E, Prassopoulos V, Andreou J: The impact of FDG-PET/ /CT on the management of breast cancer patients with elevated tumor markers and negative or equivocal conventional imaging modalities. Nucl Med Commun 2011, 32, 85-90.

[8] Evangelista L, Baretta Z, Vinante L, Cervino AR, Gregianin M, Ghiotto C, Bozza F, Saladini G: Could the serial determination of Ca15.3 serum improve the diagnostic accuracy of PET/CT?: Results from small population with previous breast cancer. Ann Nucl Med 2011, 25, 469-477.

[9] Gradishar WJ, Anderson BO, Blair SL, Burstein HJ, Cyr A, Elias AD, Farrar WB, Forero A, Giordano SH, Goldstein LJ, Hayes DF, Hudis CA, Isakoff SJ, Ljung BM, Marcom PK, Mayer IA, McCormick B, Miller RS, Pegram M, Pierce LJ, Reed EC, Salerno KE, Schwartzberg LS, Smith ML, Soliman H, Somlo G, Ward JH, Wolff AC, Zellars R, Shead DA, Kumar R: National Comprehensive Cancer Network Breast Cancer Panel. Breast Cancer v. 3.2014. J Natl Compr Canc Netw 2014, 12, 542-590. 
[10] Goldhirsch A, Glick JH, Gelber RD, Coates AS, Senn HJ: Meeting highlights: International Consensus Panel on the Treatment of Primary Breast Cancer. Seventh International Conference on Adjuvant Therapy of Primary Breast Cancer. J Clin Oncol 2001, 19, 3817-3827.

[11] Rastogi P, Anderson SJ, Bear HD, Geyer CE, Kahlenberg MS, Robidoux A, Margolese RG, Hoehn JL, Vogel VG, Dakhil SR, Tamkus D, King KM, Pajon ER, Wright MJ, Robert J, Paik S, Mamounas EP, Wolmark N: Preoperative chemotherapy: updates of National Surgical Adjuvant Breast and Bowel Project Protocols B-18 and B-27. J Clin Oncol 2008, 26, 778-785.

[12] Bear HD, Anderson S, Smith RE, Geyer CE Jr, Mamounas EP, Fisher B, Brown AM, Robidoux A, Margolese R, Kahlenberg MS, Paik S, Soran A, Wickerham DL, Wolmark N: Sequential preoperative or postoperative docetaxel added to preoperative doxorubicin plus cyclophosphamide for operable breast cancer:National Surgical Adjuvant Breast and Bowel Project Protocol B-27. J Clin Oncol 2006, 24, 2019-2027.

[13] Duch J, Fuster D, Muñoz M, Fernández PL, Paredes P, Fontanillas M, Guzmán F, Rubí S, Lomeña FJ, Pons F: 18F-FDG PET/CT for early prediction of response to neoadjuvant chemotherapy in breast cancer. Euro J Nucl Med Mol Imaging 2009, 36, 1551-1557.

[14] Kumar A, Kumar R, Seenu V, Gupta SD, Chawla M, Malhotra A, Mehta SN: The role of 18F-FDG PET/CT in evaluation of early response to neoadjuvant chemotherapy in patients with locally advanced breast cancer. Euro Radiol 2009, 19, 1347-1357.

[15] Schwarz-Dose J, Untch M, Tiling R, Sassen S, Mahner S, Kahlert S, Harbeck N, Lebeau A, Brenner W, Schwaiger M, Jaenicke F, Avril N: Monitoring primary systemic therapy of large and locally advanced breast cancer by using sequential positron emission tomography imaging with [18F]fluorodeoxyglucose. J Clin Oncol 2009, $27,535-541$.

[16] Andrade WP, Lima EN, Osório CA, do Socorro Maciel M, Baiocchi G, Bitencourt AG, Fanelli MF, Damascena AS, Soares FA: Can FDG-PET/CT predict early response to neoadjuvant chemotherapy in breast cancer? Eur J Surg Oncol 2013, 39, 1358-1363.

[17] Kim T, Kang DK, An YS, Yim H, Jung YS, Kim KS, Kang SY, Kim TH: Utility of MRI and PET/CT after neoadjuvant chemotherapy in breast cancer patients: correlation with pathological response grading system based on tumor cellularity. Acta Radiol 2014, 55, 399-408.

[18] Loo CE, Straver ME, Rodenhuis S, Muller SH, Wesseling J, Vrancken Peeters MJ, Gilhuijs KG: Magnetic resonance imaging response monitoring of breast cancer during neoadjuvant chemotherapy: Relevance of breast cancer subtype. J Clin Oncol 2011, 29, 660-666.

[19] Dehdashti F, Flanagan FL, Mortimer JE, Katzenellenbogen JA, Welch MJ, Siegel BA: Positron emission tomographic assessment of "metabolic flare" to predict response of metastatic breast cancer to antiestrogen therapy. Euro J Nucl Med 1999, 26, 51-56.

[20] Mortimer J, Dehdashti F, Siegel B, Trinkaus K, Katzenellenbogen J, Welch M: Metabolic flare: indicator of hormone responsiveness in advanced breast cancer. J Clin Oncol 2001, 19, 2797-2803.

[21] Mortazavi-Jehanno N, Giraudet AL, Champion L, Lerebours F, Le Stanc E, Edeline V, Madar O, Bellet D, Pecking AP, Alberini JL: Assessment of response to endocrine therapy using FDG PET/CT in metastatic breast cancer: a pilot study. Euro J Nucl Med Mol Imaging 2012, 39, 450-460.

[22] Huyge V, Garcia C, Alexiou J, Ameye L, Vanderlinden B, Lemort M, Bergmann P, Awada A, Body JJ, Flamen P: Heterogeneity of metabolic response to systemic therapy in metastatic breast cancer patients. J Clin Oncol 2010, $22,818-827$.

[23] García García-Esquinas M, García-Sáenz JA, Arrazola García J, Enrique Fuentes Ferrer M, Furió V, Rodriguez Rey C, Román JM, Carreras Delgado JL: 18F-FDG PET-CT imaging in the neoadjuvant setting for stages II-III breast cancer: association of locoregional SUVmax with classical prognostic factors. Euro J Nucl Med Mol Imaging $2014,58,66-73$.

[24] García Vicente AM, Soriano Castrejón A, Cruz Mora MA, González Ageitos A, Muñoz Sánchez Mdel M, León Martín A, Espinosa Aunión R, Relea Calatayud F, Muñoz Madero V, Chacón López-Muñiz I, Cordero García JM, Jiménez Londoño GA: Semi-quantitative lymph node assessment of (18)F-FDG PET/CT in locally advanced breast cancer: correlation with biological prognostic factors. Euro J Nucl Med Mol Imaging 2013, 40, 72-79.

[25] Heudel P, Cimarelli S, Montella A, Bouteille C, Mognetti T: Value of PET-FDG in primary breast cancer based on histopathological and immunohistochemical prognostic factors. Int J Clin Oncol 2010, 15, 588-593.

[26] Keam B, Im SA, Koh Y, Han SW, Oh DY, Cho N, Kim JH, Han W, Kang KW, Moon WK, Kim TY, Park IA, Noh DY, Chung JK, Bang YJ: Early metabolic response using FDG PET/CT and molecular phenotypes of breast cancer treated with neoadjuvant chemotherapy. BMC Cancer 2011, 11, 452.

[27] Martoni AA, Zamagni C, Quercia S, Rosati M, Cacciari N, Bernardi A, Musto A, Fanti S, Santini D, Taffurelli M: Early (18)F-2-fluoro-2-deoxy-d-glucose positron emission tomography may identify a subset of patients with estrogen receptor-positive breast cancer who will not respond optimally to preoperative chemotherapy. Cancer 2010, 116, 805-813.

[28] Zucchini G, Quercia S, Zamagni C, Santini D, Taffurelli M, Fanti S, Martoni AA: Potential utility of early metabolic response by $18 \mathrm{~F}$-2-fluoro-2-deoxy-D-glucose-positron emission tomography/computed tomography in a selected group of breast cancer patients receiving preoperative chemotherapy. Eur J Cancer 2013, 49, 1539-1545.

[29] Koolen BB, Pengel KE, Wesseling J, Vogel WV, Vrancken Peeters MJ, Vincent AD, Gilhuijs KG, Rodenhuis S, Rutgers EJ, Valdés Olmos RA: FDG PET/CT during neoadjuvant chemotherapy may predict response in ER-positive/HER2-negative and triple negative, but not in HER2-positive breast cancer. Breast 2013, 22, 691-697. 
[30] Shields AF, Grierson JR, Dohmen BM, Machulla HJ, Stayanoff JC, Lawhorn-Crews JM, Obradovich JE, Muzik O, Mangner TJ: Imaging proliferation in vivo with [F-18]FLT and positron emission tomography. Nat Med 1998, 4, 1334-1336.

[31] Been LB, Elsinga PH, de Vries J, Cobben DC, Jager PL, Hoekstra HJ, Suurmeijer AJ: Positron emission tomography in patients with breast cancer using (18)F-3'-deoxy-3'-fluoro-l-thymidine ((18)F-FLT) - a pilot study. Eur J Surg Oncol 2006, 32, 39-43.

[32] Pio BS, Park CK, Pietras R, Hsueh WA, Satyamurthy N, Pegram MD, Czernin J, Phelps ME, Silverman DH: Usefulness of 3'-[F-18]fluoro-3'-deoxythymidine with positron emission tomography in predicting breast cancer response to therapy. Mol Imaging Biol 2006, 8, 36-42.

[33] Contractor K, Aboagye EO, Jacob J, Challapalli A, Coombes RC, Stebbing J: Monitoring early response to taxane therapy in advanced breast cancer with circulating tumor cells and [(18)F] 3-deoxy-3 -fluorothymidine PET: a pilot study. Biomarkers Med 2012, 6, 231-233.

[34] Hospers GA, Helmond FA, de Vries EG, Dierckx RA, de Vries EF: PET imaging of steroid receptor expression in breast and prostate cancer. Curr Pharm Des 2008, 14, 3020-3032.

[35] Dehdashti F, Laforest R, Gao F, Aft RL, Dence CS, Zhou D, Shoghi KI, Siegel BA, Katzenellenbogen JA, Welch MJ: Assessment of progesterone receptors in breast carcinoma by PET with 21-18F-fluoro-16alpha,17alpha[(R)-(1'-alpha-furylmethylidene)dioxy]-19-norpregn- 4-ene-3,20-dione. J Nucl Med 2012, 53, 363-370.

[36] Dijkers EC, Oude Munnink TH, Kosterink JG, Brouwers AH, Jager PL, de Jong JR, van Dongen GA, Schröder CP, Lub-de Hooge MN, de Vries EG: Biodistribution of 89Zr-trastuzumab and PET imaging of HER2positive lesions in patients with metastatic breast cancer. Clin Pharmacol Ther 2010, 87, 586-592.

[37] Piccardo A, Altrinetti V, Bacigalupo L, Puntoni M, Biscaldi E, Gozza A, Cabria M, Iacozzi M, Pasa A, Morbelli S, Villavecchia G, DeCensi A: Detection of metastatic bone lesions in breast cancer patients: fused (18)F-FluoridePET/MDCT has higher accuracy than MDCT. Preliminary experience. Eur J Radiol 2012, 81, 2632-2638.

[38] Iagaru A, Mittra E, Mosci C, Dick DW, Sathekge M, Prakash V, Iyer V, Lapa P, Isidoro J, de Lima JM, Gambhir SS: Combined 18F-fluoride and 18F-FDG PET/CT scanning for evaluation of malignancy: Results of an international multicenter trial. J Nucl Med 2013, 54, 176-183.

\section{Address for correspondence:}

Zhanhong Chen

Department of Chemotherapy Center

Zhejiang Cancer Hospital

38 Guangji Road, Hangzhou

Zhejiang 310022

China

E-mail: leilei1241@163.com

Conflict of interest: None declared

Received: 15.06 .2014

Revised: 22.07.2014

Accepted: 5.12.2014 